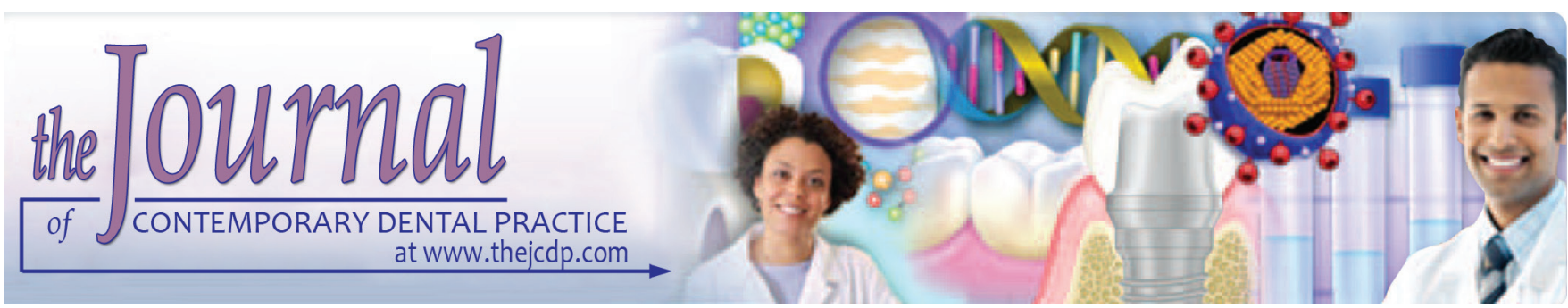

\title{
Bilateral Condylar Hyperplasia-Nonsurgical Management: A Clinical Report
}

\author{
${ }^{1}$ Adrian Seto, ${ }^{2}$ Michael G Botelho, ${ }^{3}$ Edward HT Ho, ${ }^{4}$ Nithya Jagannathan
}

\begin{abstract}
Background: Patients present with malocclusions in the form of bilateral open bite with functional and esthetic challenges. These are particularly so whereby these are acquired through growth anomalies that create a change in the occlusal status whereby a patient has to adapt to the challenge of the occlusal change. While surgical intervention is a considered option to correct such changes, not all patients are willing to endure the consequences of this intervention and as such this report presents a conservative minimally invasive approach.
\end{abstract}

Aim: This clinical report demonstrates a nonsurgical approach in the management of a patient with bilateral metabolically active condylar hyperplasia.

Case report: The patient presented with a bilateral open bite with occlusal contacts only present on her second molars. The severity of the open bite was reported to be recently progressing and getting worse. This condition had impaired her chewing function and quality of life over an 8-year period. Bone scintigraphy was performed and metabolically active bilateral condylar hyperplasia was diagnosed. The patient refused surgical intervention and instead a conservative overlay denture was prescribed, which successfully restored her function and esthetics.

Conclusion: Removable overlay dentures can be a relatively simple and effective treatment option for patients presenting with newly acquired bilateral open bites to give a functional and esthetic outcome.

Clinical significance: This clinical report shows the conservative management of bilateral condylar hyperplasia with a simple removable overlay appliance. The occlusion has stabilized and the patient functions well.

\footnotetext{
${ }^{1,3}$ Department of Prosthodontics, Hong Kong Sanatorium and Hospital, Happy Valley, Hong Kong, People's Republic of China

${ }^{2,4}$ Department of Prosthodontics, Faculty of Dentistry, The University of Hong Kong, The Prince Philip Dental Hospital, Sai Ying Pun, Hong Kong, People's Republic of China

Corresponding Author: Michael G Botelho, Department of Prosthodontics, The University of Hong Kong, Room 3B19, The Prince Philip Dental Hospital, 34, Hospital Road, Sai Ying Pun Hong Kong, People's Republic of China, e-mail: botelho@hku.hk
}

Keywords: Bone scintigraphy, Condylar hyperplasia, Open bite, Overlay denture.

How to cite this article: Seto A, Botelho MG, Ho EHT, Jagannathan N. Bilateral Condylar Hyperplasia-Nonsurgical Management: A Clinical Report. J Contemp Dent Pract 2018;19(4):463-467.

Source of support: Nil

Conflict of interest: None

\section{BACKGROUND}

Condylar hyperplasia is a pathological condition characterized by overgrowth of the condylar process. ${ }^{1}$ This can affect the patient by an increasing open bite, and thus leading to occlusal instability and deficiency in masticatory function. This condition presents clinically with temporomandibular joint (TMJ) pain and radiographically elongation of the condylar neck or an enlargement of the condyle, or both may be present. ${ }^{2}$ Shifting of the mandibular midline to the relatively less affected side can lead to variable abnormal mandibular or facial symmetry. In case of increasing mandible asymmetry, a stable result can be achieved by surgical intervention of a high condylectomy which involves shaving of the condylar head followed by orthognathic surgery. ${ }^{3}$ However, patients having facial asymmetry with intact occlusion and ceased condylar growth can be treated with mandibular inferior border osteotomy and facial contouring based on the severity of asymmetry. While surgery is considered the primary mode of treatment, this case report discusses on a conservative approach in treating bilateral condylar hyperplasia. ${ }^{4}$

\section{CASE REPORT}

This clinical report documents a 63-year-old Chinese female who was suffering from a progressing open bite with class III American College of Prosthodontists 
Prosthodontic Diagnostic Index and partial edentulism. ${ }^{5}$ The patient presented with an open bite which was reported as increasing in rate over the last 2-year period as well as impaired masticatory function. The patient had implemented a change in her dietary habit to include softer food to relieve TMJ pain and improve chewing efficiency.

\section{Clinical Examination}

The patient was referred to the Oral and Maxillofacial Discipline, Faculty of Dentistry at the University of Hong Kong, for evaluation and treatment. Clinically, she had a bilateral anterior open bite extending from the right second premolar to left second premolar with a moderate cross bite on both sides with an Angle's class I dental relationship. The upper right first molar and lower left second molars had a poor prognosis due to advanced periodontitis and were planned for extraction (Fig. 1). The patient reported bilateral pain in the TMJ region during eating.

\section{Medical and Dental History}

There was no significant medical or dental history.

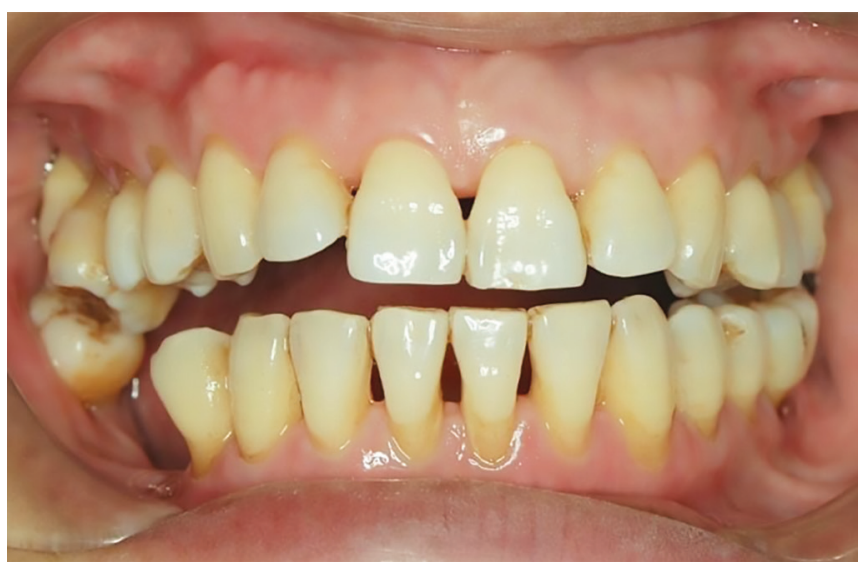

Fig. 1: Pretreatment view in centric occlusion showing the extent of the open bite

\section{Special Investigation}

Condylar hyperplasia was suspected and special investigations were performed to examine the patient metabolic rate and condylar growth activity. A thyroid hormone imbalance was suspected and blood tests were prescribed; however, thyroid-stimulating hormone (T3 and T4) was at a normal level, suggesting a normal metabolic rate. After this, further investigations were performed to determine the condylar activity using a bone scintigraphy after an injection of $25 \mathrm{mCi}$ of radiotracer technetium-methylene diphosphonate. When the radiotracer breaks down, it emits radiation and the sensor detects the radiation that is accumulated and abnormal activity shows "hot spots" if increase in accumulation of radioactive material occurs. ${ }^{6}$ The result confirmed the provisional diagnosis of metabolically active bilateral condylar hyperplasia (Fig. 2).

\section{Treatment Plan}

Surgical intervention was suggested but the patient declined this. She was then referred to the oral rehabilitation discipline, within the faculty. With careful consideration of the clinical manifestation and patient's functional need, a treatment plan was developed with objectives of restoration of masticatory function and observation of occlusal change. A noninvasive and reversible treatment approach was decided by way of fabricating an overlay denture to restore masticatory function.

\section{Pretreatment Preparation}

The patient's study models were mounted in centric occlusion on a semi-adjustable articulator (Denar Mark II Articulator) which revealed isolated contacts on the last two pairs of molars. Occlusal interference was seen on both sides during lateral excursion. Two sets of duplicate models were mounted on the semi-adjustable articulator. On the first set, an occlusal adjustment was performed on the interfering molar pairs to bring the occlusal surfaces
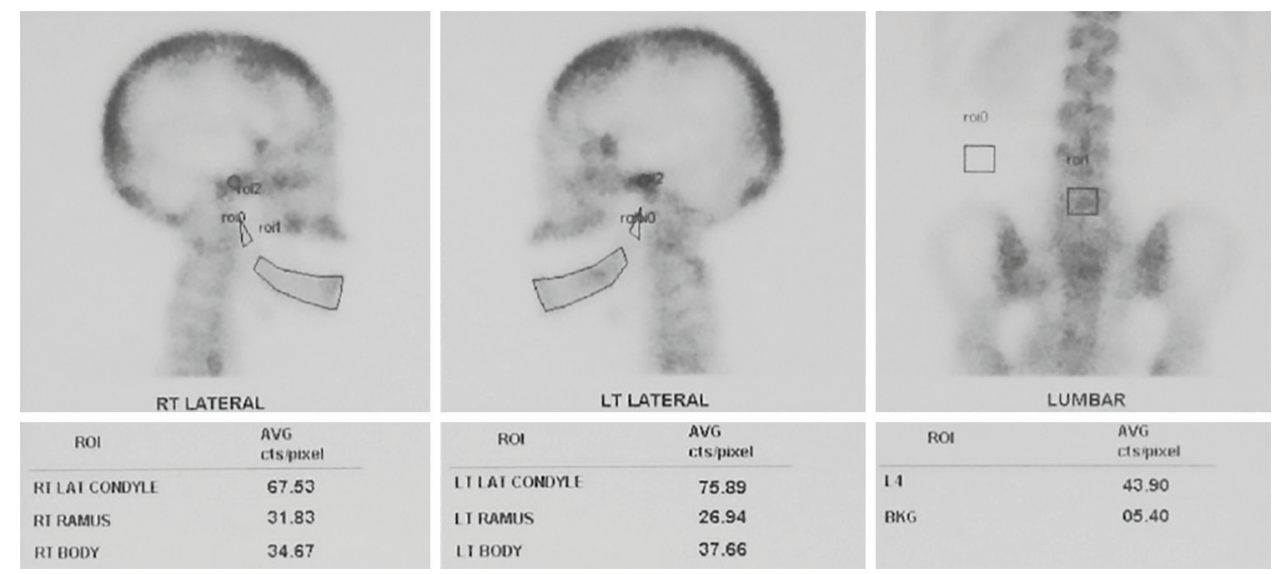

Fig. 2: Mandibular bone scintigraphy showing hotspots for metabolically active condyles 


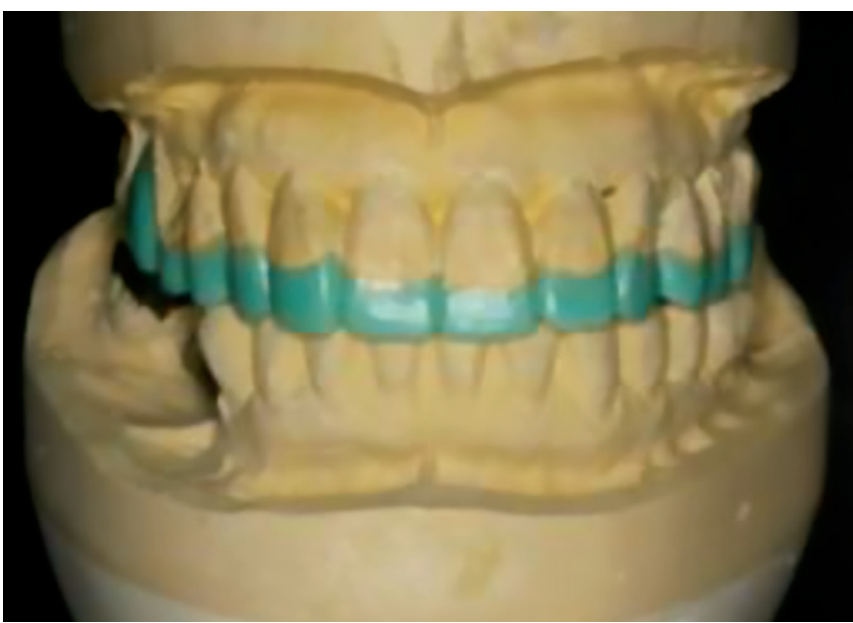

Fig. 3: Diagnostic wax-up in centric occlusion to show patient prosthesis outcome

together; however, a good cuspal interdigitation was not achieved. Furthermore, the occlusal adjustment would have required $3 \mathrm{~mm}$ tooth surface loss which would involve elective endodontic treatment or extraction. On the second set, a diagnostic wax-up was performed to examine conservative occlusal reconstruction. Group function was planned on both sides (Fig. 3).

\section{Treatment Procedure}

After discussion with the patient, she did not want any tooth tissue removal or additional tooth extraction, and therefore, a conservative option was followed. A maxillary overlay denture (Fig. 4) was made according to the diagnostic wax-up. Intercuspal occlusal contacts were planned from the canines distally on all the posterior teeth, with the incisors in light occlusion. Direct composite resin (Filtek Z250 Universal Restorative, 3M ESPE) onlays were provided on the mesially tilted lower right first premolar and second molar to increase the nature of the occlusal contacts on these teeth. Group function in lateral excursion was established on both sides by careful intraoral adjustment on the acrylic overlay denture. Tooth contacts between maxillary acrylic teeth and mandibular natural teeth were checked by the holding resistance exerted on shimstock at centric occlusion.? The patient was instructed to maintain a high standard of both oral and denture hygiene and to remove the denture at night.

\section{Postoperative Course and Prognosis}

Postinsertion review after two 3-month intervals included checking of the occlusal contacts (Fig. 5). Clinical examination revealed good oral and denture hygiene was maintained. Occlusal contacts at the lower right premolars were lost as determined by shimstock

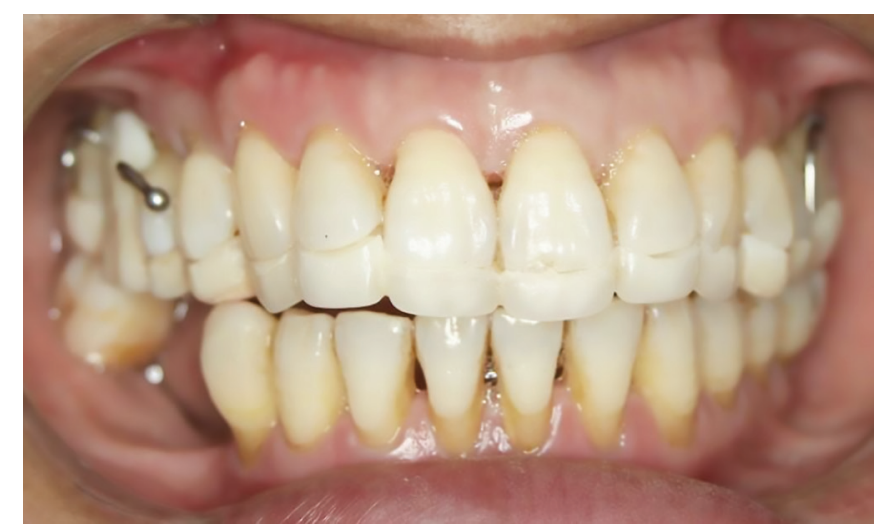

Fig. 4: Heat-cured acrylic overlay denture based on diagnostic wax-up in rest position

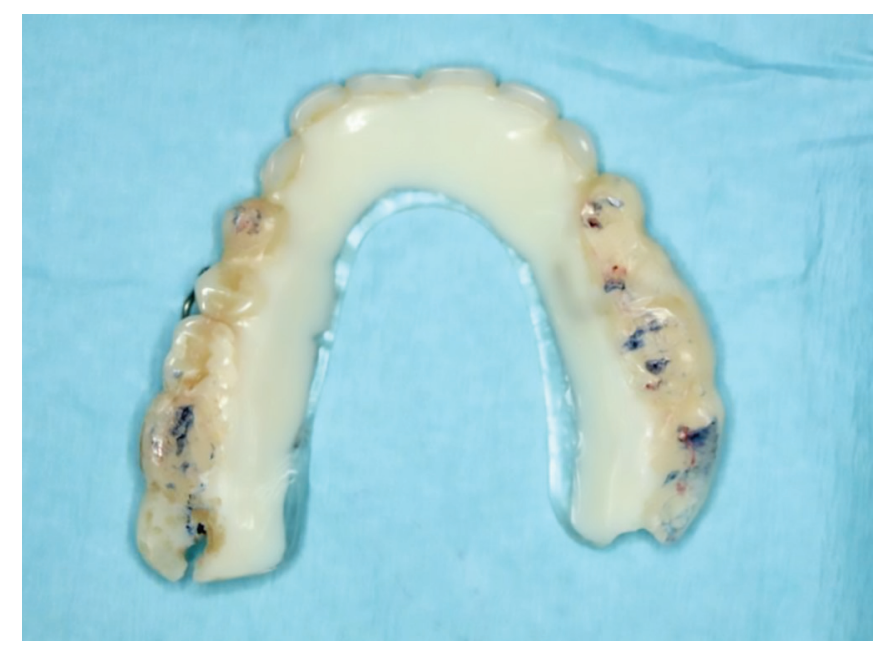

Fig. 5: Overlay denture showing occlusal contacts of all posterior teeth in centric occlusion

and articulating paper. No bruxing or wearing was apparent in this area of the overlay denture, which suggested that mandibular hyperplasia had not ceased as the tooth contacts were lost due to continued condylar growth. Cold cure acrylic (GC Unifast TRAD, GC Corporation) was added to the denture base to re-establish tooth contacts that held shimstock. At this appointment, the patient reported that TMJ pain during eating had subsided completely. She could now chew comfortably and with improved efficiency.

It was decided for the patient to wear the overlay denture for at least 1 year and to review the occlusion at 3-month intervals and to re-establish occlusal contacts if by checking the occlusion to maintain her function without the invasive procedures. Subsequent regular review appointments revealed that there was minimal change in occlusion on the overlay denture. Occasionally, there was one area on the overlay denture that did lose occluding contacts which were opposing the lower right premolars. This required the addition of cold cure acrylic to the overlay to reestablish the occlusal contacts. After 18 months monitoring and reviews, the patient perceived the 
change in the occlusion had stopped and there were no significant changes in the occlusal contacts on the overlay denture. At this time, the review interval was increased to 6-month time with no significant change.

\section{DISCUSSION}

Condylar hyperplasia is a rare condition first described by Robert Adams in 1986 and characterized by uncontrolled prechondroblastic cellular activity at the head or neck of condyle, resulting in progressive and independent growth of the condyles. ${ }^{8}$

While the pathogenesis of condylar hyperplasia remains unclear, a range of intrinsic and extrinsic etiological factors have been reported. These include trauma followed by hyperplasia, genetic or hormonal causes, intrauterine changes, joint infection, hypervascularity of condylar tissues and increase in functional loading of TMJ. ${ }^{9,10}$

This condition appears commonly in adolescents in the age range between 10 and 30 years. However, the age of onset is variable as it has been reported in patients over 55 years, and hence, it is not possible to define an exact age range. It is often associated with female predilection as an increased number of estrogen receptors has been reported in the TMJ of females. ${ }^{11,12}$

Obwegeser and Makek ${ }^{13}$ classified condylar hyperplasia into three major types: hemimandibular hyperplasia causing asymmetry in vertical plane, hemimandibular elongation resulting in asymmetry along the transverse plane, and condylar hyperplasia as a combination of both and can manifest as hybrid unilateral and combined bilateral. While the occurrence of bilateral condylar hyperplasia is rare, Wolford et $\mathrm{al}^{14}$ in a study of 2,914 patients, reported on the possibility of occurrence of this condition. Facial asymmetry is the most common manifestation of condylar hyperplasia, and the increase in condylar growth leads to articular dysfunction and deviation of the jaw toward the contralateral side based on the degree of variation of condylar growth between both sides. This condition is also associated with occlusal disharmony which would result in esthetic concerns and functional alterations. ${ }^{15,16}$

The initial steps in diagnosing condylar hyperplasia include documentation of clinical findings, study models, and radiographic images, such as intraoral and extraoral photographs, panoramic images, lateral cephalogram, and computed tomography. Diagnosing active condylar growth is essential to formulate a treatment plan and mandibular condyle growth is best assessed by skeleton bone scintigraphy with single-photon emission computed tomography of the head. ${ }^{17,18}$ However, due to the dosage of radiation with bone scintigraphy, routine use is not recommended. However, if it is planned to provide a full-mouth fixed prosthodontic restorative solution to the open bite, it may be deemed appropriate to determine the cessation of condylar hyperplasia before undertaking such extensive treatment. In this case, a removable prosthesis approach was considered the long-term solution.

The treatment of facial asymmetry resulting from condylar hyperplasia is variable, and determining the activity of condylar growth is essential to determine the appropriate treatment plan. The age of the patient, severity of asymmetry, and resulting malocclusion and TMJ symptoms are other factors to be considered when planning treatment. Initially, observation and symptomatic management of the condition is the first consideration. If increasing and getting severe, control of the condylar growth by surgical intervention may be considered to balance facial harmony. In patients with ongoing condylar hyperplasia and mandibular asymmetry, a two-phase surgical orthodontic approach is usually considered a necessary and a successful treatment protocol. ${ }^{3,19}$ This usually involves the removal of condylar cartilage to inhibit growth. Thus, progressive mandibular growth ceases followed by possible orthognathic surgery. ${ }^{20,21}$ However, when the condyle growth is ceased, the condition is treated as facial asymmetry which is treated by orthodontic or surgical orthognathic approach.

In this clinical report, the progression of increasing open bite appears to have slowed down. A surgical approach is considered an established procedure to inhibit hyperplasia, but the patient opted for noninvasive approach to restore her occlusion and chewing function. However, recent review appointments at 18 months after insertion of overlay denture revealed some tooth contact loss on the prosthesis to the lower premolar teeth. To reevaluate any change and maintain posterior tooth contacts, frequent review and occlusal adjustment on the overlay denture are necessary.

The above-mentioned treatment approach has provided a conservative noninvasive and functional restorative solution to a patient presenting with bilateral condylar hyperplasia who did not want a surgical intervention. The patient was satisfied with the esthetic and functional outcome but requires ongoing monitoring and addition to the denture to maintain the occlusion. A surgical intervention could still be considered later if indicated and with patient consent.

\section{CONCLUSION}

This case report demonstrates a reversible cost-effective treatment approach to solve both functional and esthetic needs for a patient presenting with a recently acquired bilateral open bite. Ongoing maintenance will be required and definitive treatment options can still be considered at a later time if patient driven. 


\section{CLINICAL SIGNIFICANCE}

While the treatment of bilateral condylar hyperplasia may be effectively managed through surgical interventions, this case report discusses on conservative and noninvasive management of this condition using a removable overlay appliance.

\section{REFERENCES}

1. Obwegeser, HL. Condylar hyperactivity. In: Obwegeser HL, editor. Mandibular growth anomalies. Berlin: Springer-Verlag; 2001. pp. 139-144.

2. Egyedi P. Aetiology of condylar hyperplasia. Aust Dent J 1969 Feb;14(1):12-17.

3. Deleurant Y, Zimmermann A, Peltomäki T. Hemimandibular elongation: treatment and long-term follow-up. Orthod Craniofac Res 2008 Aug;11(3):172-179.

4. Olate S, Netto HD, Rodriguez-Chessa J, Alister JP, AlbergariaBarbosa JD, Moraes MD. Mandibular condylar hyperplasia: a review of diagnosis and treatment protocol. Int J Clin Exp Med 2013 Sep;6(9):727-737.

5. McGarry TJ, Nimmo A, Skiba JF, Ahlstrom RH, Smith CR, Koumjian JH, Arbree NS. Classification system for partial edentulism. J Prosthodont 2002 Sep;11(3):181-193.

6. Newberg, A. Bone scans. In: Pretorius ES, Solomon JA, editors. Radiology secrets plus. 3rd ed. Chapter 54. Philadelphia (PA): Elsevier Mosby; 2010.

7. Harper KA, Setchell DJ. The use of shim stock to assess occlusal contacts: a laboratory study. Int J Prosthodont 2002 Jul-Aug;15(4):347-352.

8. Robinson J, O'Brien A, Chen J, Wadhwa S. Progenitor cells of the mandibular condylar cartilage. Curr Mol Biol Rep 2015 Sep;1(3):110-114.

9. Pereira-Santos D, De Melo WM, Souza FA, de Moura WL, Cravinhos JC. High condylectomy procedure: a valuable resource for surgical management of the mandibular condylar hyperplasia. J Craniofac Surg 2013 Jul;24(4):1451-1453.

10. Ahn SJ, Lee SP, Nahm DS. Relationship between temporomandibular joint internal derangement and facial asymmetry in women. Am J Orthod Dentofacial Orthop 2005 Nov;128(5):583-591.

11. Ribeiro-Dasilva MC, Peres LineSR, LemeGodoy dos Santos MC, Arthuri MT, Hou W, Fillingim RB, Rizzatti Barbosa CM. Estrogen receptor-alpha polymorphisms and predisposition to TMJ disorder. J Pain 2009 May;10(5):527-533.

12. Mutoh Y, Ohashi Y, Uchiyama N, Terada K, Hanada K, Sasaki F. Three-dimensional analysis of condylar hyperplasia with computed tomography. J Craniomaxillofac Surg 1991 Feb;19(2):49-55.

13. Obwegeser HL, Makek MS. Hemimandibular hyperplasia hemimandibular elongation. J Maxillofac Surg 1986 Aug;14(4): 183-208.

14. Wolford LM, Movahed R, Perez DE. A classification system for conditions causing condylar hyperplasia. J Oral Maxillofac Surg 2014 Mar;72(3):567-595.

15. Posnick, JC. Asymmetric mandibular excess growth patterns. In: Posnick JC, editor. Orthognathic surgery: principles and practice. St. Louis (MO): Elsevier; 2014. pp. 807-865.

16. Wolford LM, Morales-Ryan CA, García-Morales P, Perez D. Surgical management of mandibular condylar hyperplasia Type 1. Proc (Bayl Univ Med Cent) 2009 Oct;22(4):321-329.

17. Laverick S, Bounds G, Wong WL. [18F]-fluoride positron emission tomography for imaging condylar hyperplasia. Br J Oral Maxillofac Surg 2009 Apr;47(3):196-199.

18. Alyamani A, Abuzinada S. Management of patients with condylar hyperplasia: a diverse experience with 18 patients. Ann Maxillofac Surg 2012 Jan;2(1):17-23.

19. Bouchard, C.; Lawler, M.; Kaban, LB.; Troulis, MJ. Minimally invasive orthognathic and condylar surgery. In: Guttenberg SA, editor. Cosmesis of the mouth, face and jaws. West Sussex: John Wiley \& Sons, Inc.; 2013. pp. 308-317.

20. Lippold C, Kruse-Losler B, Danesh G, Joos U, Meyer U. Treatment of hemimandibular hyperplasia: The biological basis of condylectomy. Br J Oral Maxillofac Surg 2007 Jul;45(5):353-360.

21. Wolford LM, Mehra P, Reiche-Fischel O, Morales-Ryan CA, García-Morales P. Efficacy of high condylectomy for management of condylar hyperplasia. Am J Orthod Dentofacial Orthop 2002 Feb;121(2):136-150. 